\title{
La formación universitaria y su relación con el comportamiento ambiental de los estudiantes del CUNSURORI, USAC, Jalapa.
}

University education and its relationship with the environmental behavior of students in CUNSURORI, USAC, Jalapa.

Jerson Martínez-Castro ${ }^{1}$ https://doi.org/10.46954/librosfahusac.9

\section{Resumen}

OBJETIVO: relacionar la formación universitaria con el comportamiento ambiental de los estudiantes en las distintas carreras del Centro Universitario de Sur Oriente-CUNSURORIde la Universidad de San Carlos de Guatemala -USAC-, con sede en la ciudad de Jalapa. METODO: se tomó en cuenta el enfoque del paradigma neopositivista, con un diseño mixto de estudio cuantitativo y cualitativo. El método cuantitativo fue no experimental y se realizaron pruebas de normalidad, consistencia interna, validez, correlaciones y análisis de varianza, no paramétrico, de medias y factorial exploratorio para las escalas NEP-R y ECE. El método cualitativo conllevó un análisis fenomenológico para autoridades del CUNSURORI con entrevista semiestructurada para realizar una codificación abierta, axial y selectiva. RESULTADOS: las escalas mencionadas presentaron una consistencia interna, por medio del alfa de Cronbach, en promedio de 0.674 por lo que efectivamente miden la preocupación y el comportamiento ambiental. Para la validación del constructo se realizó análisis factorial exploratorio con la prueba KMO y se obtuvo un valor 
de 0.681 y 0.775 para la escala NEP-R y ECE, respectivamente. En lo cualitativo hay poca preocupación por el tema ambiental. CONCLUSIÓN: el deterioro del ambiente y el uso inapropiado de los recursos naturales es una preocupación creciente en las sociedades del mundo. La ciencia busca explicaciones y propuestas que permitan minimizar el deterioro creciente del ambiente. Las diferencias del comportamiento ambiental de los estudiantes al ingreso y egreso universitario no son significativas, además, es mínima la importancia que le proveen los responsables a contenidos y actividades ambientales.

Palabras clave: formación universitaria, comportamiento ambiental, NEP-R, ECS, centro universitario, carreras profesionales, estudiantes.

\section{Abstract}

OBJECTIVE: to relate university education to the environmental behavior of students in the different careers of the South East University Center -CUNSURORI - of the Universidad de San Carlos de Guatemala -USAC -, based in the city of Jalapa. METHOD: the neopositivist paradigm approach was taken into account, with a mixed quantitative and qualitative study design. The quantitative method was non-experimental and tests of normality, internal consistency, validity, correlations and analysis of variance, non-parametric, mean and exploratory factorial were performed for the NEP-R and ECE scales. The qualitative method involved a phenomenological analysis for CUNSURORI authorities with a semi-structured interview to perform open, axial and selective coding. RESULTS: the scales mentioned presented an internal consistency, by means of Cronbach's alpha, on average of 0.674 so that they effectively measure environmental concern and behavior. For the validation of the construct, exploratory factor analysis was performed with the KMO test and a value of 0681 and 0.775 was obtained for the NEP-R and ECE scale, respectively. In terms of quality, there is little concern for the environmental issue. CONCLUSION: the deterioration of the environment and the inappropriate use of natural resources 
is a growing concern in the societies of the world. Science seeks explanations and proposals to minimize the increasing deterioration of the environment. The differences in the environmental behavior of students at university entrance and exit are not significant, in addition, the importance that those responsible provide to environmental contents and activities is minimal.

Keywords: University education, environmental behavior, NEP-R, ECS, university center, professional careers, students

\section{Introducción}

A través de la educación los individuos desarrollan las capacidades de analizar, comparar y razonar sobre los diferentes aspectos de su realidad. La formación secuencial en la universidad permite desarrollar destrezas y ser conscientes de los diferentes aspectos que le rodean y que definen su entorno. Se desarrolló una investigación con el objetivo de relacionar la formación profesional universitaria y el comportamiento ambiental de los estudiantes del Centro Universitario de Sur Oriente -CUNSURORI- de la USAC con sede en Jalapa, donde se imparten seis carreras profesionales: Administración de Empresas, Agronomía, Ciencias Jurídicas y Sociales, Pedagogía, Trabajo Social y Zootecnia. Para realizar dicho estudio se realizaron encuestas y entrevistas durante los años 2019 y 2020 , previa a las limitaciones por la pandemia de COVI-19.

La investigación se basó en el constructo formación universitaria y comportamiento ambiental. Un constructo se define como aquel concepto teórico que tiene un referente práctico que se refiere a un fenómeno de la realidad. De acuerdo a Mendoza y Garza (2009), el constructo representa una variable cuantitativa que está inmersa dentro de una hipótesis o modelo teórico. Se analizó si, a través de los ciclos de formación de cada carrera, el comportamiento respecto al tema ambiental cambia positivamente. La mayoría de los ciclos de las están comprendidos desde el primero hasta el 
décimo, en un rango de cinco años, durante los cuales se deben aprobar entre cuarenta y tres y sesenta y un cursos, dependiendo de la carrera que se trate.

Los pensa de estudios integran diferentes cursos que comprenden una formación del área básica, social humanística, técnica y profesional. A medida que los estudiantes aprueban los cursos de su carrera, adquieren capacidades, habilidades, destrezas y competencias en su campo profesional. Una formación universitaria integral les permite una mayor visión de su realidad y del ámbito profesional que se forman. Además, desarrollan el análisis y raciocinio sobre diferentes temas sociales, económicos, humanísticos y ambientales. Toda esta formación moldea el comportamiento de los estudiantes hacia los diferentes temas de su educación universitaria (Miranda, 2013 y Tyler, 1986).

Esta investigación permitió relacionar si la formación integral que se recibe en la universidad permite desarrollar comportamientos y preocupación en torno a la problemática ambiental. Hay varios autores que hacen afirmaciones opuestas en torno a esto, por ejemplo, Acosta-Martínez et al. (2001) opina que el conocimiento ambiental no influye sobre la conducta ambiental de las personas. Zapata \& Castrechini (2011) consideran que la personalidad no condiciona la conducta ambiental de las personas. Por otro lado, Tyler (1986) indica que la educación favorece cambios de comportamiento, sentimientos y pensamientos para realizar acciones positivas. Se considera que los estudiantes deben recibir la información adecuada para comprender el problema, desarrollar estrategias de acción y capacitarse en criterios de sustentabilidad (Uzzell \& Rathzel, 2009). Si las personas tienen información suficiente pueden promover una conducta ambiental favorable y desarrollar motivaciones para creer y convencerse que sus acciones son efectivas (Alvarez \& Vega, 2009). Por ello, el manejo proambiental depende del conocimiento, las actitudes y el comportamiento (Isaacmárquez, et al., 2011). 
El problema ambiental es real ya que es notorio el inadecuado manejo ambiental de cualquier entorno. Al estudiar la influencia de la educación en el comportamiento ambiental de los estudiantes es oportuno determinar la influencia de algunos factores como las características sociodemográficas que poseen, distinguir cambios de comportamiento por medio de las propias percepciones ambientales de los estudiantes en torno a la naturaleza, creencias y preocupación. Así mismo, si el conocimiento ambiental es producto de la formación universitaria que reciben, si existen contenidos curriculares en las carreras universitarias que favorecen el aprendizaje ambiental. Además, si las autoridades y docentes le proveen importancia al cuidado del ambiental en la formación universitaria.

Actualmente se requiere de una educación ambiental a todo nivel, principalmente en el universitario que forma, dirige y organiza la actividad productiva, administrativa, financiera y legal de otros niveles profesionales y educativos de la sociedad. Los individuos serán más conscientes del manejo ambiental de su entorno en la medida que tengan alguna formación al respecto, por lo que el manejo proambiental depende del conocimiento, actitudes y comportamiento (Isaac-márquez, et al., 2011). Se considera que los cambios de comportamiento dependen de la sociedad, conocimiento y creencias (Páramo, 2016), lo que hace necesario desarrollar en la sociedad mayor conciencia del problema ambiental a través de la formación, proveyendo del conocimiento necesario para ser conscientes de la realidad. El comportamiento y las actitudes pro ambientales van a depender de la estructura social, el conocimiento con que cuenta y las creencias (Páramo, 2016). La educación puede influir mucho en los cambios de actitudes en la medida que se imparta la formación adecuada.

\section{Metodología}

El estudio realizado se basó en la perspectiva epistemológica del neopositivismo. La metodología utilizada tomó en cuenta el diseño mixto, que abarca el estudio cuantitativo y cualitativo. EI diseño cuantitativo puede ser experimental y no experimental 
(Velásquez, 2017). La investigación se desarrolló bajo un diseño no experimental, el cual no trata de replicar la realidad para la manipulación de variables a manera de realizar y registrar influencia entre las mismas. El investigador se constituye en un observador del fenómeno de estudio en el contexto que se desarrolla, para luego analizarse (Navarro et al., 2017). En este tipo de diseño, el investigador recolecta datos, lo cual puede hacerlo en un momento determinado o bien realiza un estudio comparativo a través de los resultados que se le presenten en el tiempo (Bonilla, 2019).

El tipo de investigación no experimental puede ser de transversal o longitudinal (Briones, 1996; Velásquez, 2017) dependiendo del rango de tiempo en que se realizan. La investigación realizada fue transversal, pues pretendió conocer un momento determinado del tiempo, es decir, describe el fenómeno en el rango de tiempo que duró el estudio (Corona, 2016; Tamayo, 2007). En el estudio de los problemas que surgen de las interacciones humanas, pueden utilizarse tanto los métodos cualitativos como cuantitativos para resolverlos (Baena, 2017; COBAO, 2017). El método cualitativo permite hacer análisis de observaciones generales y particulares del problema en un rango de tiempo más amplio y describirlo de forma lógica con razonamiento descriptivo (Hernández-Sampieri, 2014). La investigación planteada se llevó a cabo durante el año 2019 y 2020 previo a la etapa de pandemia. Fue censada la mayoría de ciclos de las diferentes carreras del centro universitario.

El comportamiento como una característica de las personas en los estratos sociales dependen de la educación, la cual se encuentra dentro de la propia organización social, estrato económico, la cultura, en un contexto en particular (Guarisma, 2008; Reyes, 1959). El razonamiento deductivo prueba una teoría al reunir resultados de instrumentos de encuestas o censos que permiten confirmar o rechazar una teoría (Best, 1982). De manera que el razonamiento del diseño de investigación que se plantea es deductivo. 
Las investigaciones cuantitativas no experimentales de tipo transversal pueden tener diferentes tipos de alcance como el exploratorio, descriptivo, correlacional y explicativo (Hernández-Sampieri, 2014; Piloña, 2005 y Velásquez, 2017). En cuanto a la investigación correlacional, que fue la utilizada, permite encontrar las relaciones entre variables dependientes e independientes y encontrar los aspectos de causa y efecto que las caracterizan. En síntesis, la investigación realizada fue de diseño mixto, donde la parte cuantitativa es no experimental, de tipo transversal, con alcance correlacional.

La investigación analizó la formación universitaria como un factor que se relaciona con el comportamiento proambiental. Las variables que se analizaron fueron formación universitaria como variable independiente y comportamiento ambiental como variable dependiente. En la formación universitaria, las dimensiones de estudio fueron los ciclos de formación, cursos con enfoque ambiental y contenidos programáticos con contenido ambiental. El comportamiento ambiental se enfoca en las dimensiones de estudios, como lo son las creencias ecológicas, preocupación ambiental y las características sociodemográficas.

El enfoque neopositivista considera que la realidad es perceptible, notoria, medible y aunque existe, solo se puede tener un acercamiento a ella (Ramos, 2015). Esta corriente propone que no existen hechos puros por lo que todos los resultados tienen cierto grado de error. Los datos deben verificarse para crear conocimiento científico, de manera que las hipótesis deben comprobarse con las suficientes observaciones (Bernal, 2006 y Espinoza, 2010). Al analizar los fenómenos de estudio es posible de aplicar el razonamiento deductivo- inductivo y viceversa. El neopositivismo propone un lenguaje científico unificado para que sea más fácil de comprender para todos (Gutiérrez, 1992). Algunos de los principales teóricos del neopositivismo son el francés Augusto comete (1798-1857) y el austriaco británico Karl Popper (19021- 1994). 
La investigación se basó sobre la teoría de los componentes del ambiente social y natural descritos por Bifani, que los conforman la cultura, recursos naturales, organización social y base económica (Bifani, 1984). Para extraer la información en la investigación cualitativa se realizó un muestreo estratificado aleatorio con distribución porcentual entre todos los estudiantes registrados en el centro universitario. Se elaboró un instrumento que fue contestado por los estudiantes, el cual contenía diversos cuestionarios sobre información sociodemográfica, escalas para medir el comportamiento y preocupación ambiental, información ambiental de cursos. La parte cualitativa se basó en el enfoque fenomenológico y se realizó un muestreo por conveniencia y se entrevistaron a las autoridades académicas y administrativas del CUNSURORI. Al analizar la cultura hay que considerar que la misma es el resultado de la acción individual o colectiva que favorece guiar el uso de recursos naturales y la responsabilidad ambiental (Espinosa, 2015). La cultura se basa en creencia, conocimientos y valores (Motta, 1994) y puede ser moldeada por la educación (Mata-Segreda, 2004).

\section{Instrumentos}

Esta investigación representa un estudio sobre los conocimientos y percepciones de estudiantes de distintas carreras del CUNSURORI, respecto a sus percepciones sobre su conocimiento ambiental, al nuevo paradigma ecológico ambiental, la preocupación ambiental y las opiniones de las autoridades universitarias. Se analizó la formación académica universitaria y el comportamiento ambiental de los estudiantes en los distintos niveles que cursan. Se indagó si la formación académica universitaria provee a los estudiantes de información y conocimientos para reconocer la situación actual del ambiente y las posibles acciones para mitigar los efectos del deterioro. Las apreciaciones ambientales de estudiantes incluyen actitudes, preocupaciones, creencias, paradigmas, valores y puntos de vista respecto al ambiente (Isaac-márquez, et al., 2011). 
Para obtener la información pertinente, el instrumento utilizado fue un cuestionario que contenía la escala del Nuevo Paradigma Ecológico (NEP-R), la escala de la Preocupación Ambiental (ECS/ ECE), preguntas sobre características sociodemográfica y el conocimiento sobre temas ambiental que favorecen en la educación universitaria y los cursos que proveen de tal conocimiento. Además, se utilizó un cuestionario como guía de entrevista para indagar sobre la importancia que le proveen los docentes al tema ambiental. El comportamiento ambiental respecto a las creencias generales de los individuos sobre el medio ambiente fue posible medirse a través de la escala del Nuevo Paradigma Ecológico (NEP) propuesto por Dunlap, Van Liere y otros (Sanz \& Guillén, 2005). Dicha escala fue modificado por Moyano-Díaz y Palomo-Vélez, la misma representa una forma ecologista de entender las relaciones del ser humano con la naturaleza (Moyano-Díaz \& Palomo-Vélez, 2014). La escala del Nuevo Paradigma Ecológico, actualmente conocido por NEP-R, es una forma ecologista de entender las relaciones del ser humano con la naturaleza y recoge las creencias generales que las personas tienen sobre el medio ambiente.

Para medir la preocupación ambiental se utilizó la escala del mismo nombre, que se toma de la original Environmental Concern Scale (ECS) de Weigel y Weigel de 1978 y su adaptación al castellano por Aragones y Amérigo en 1991, modificado por Amérigo y Gonzales en 1996, la más reciente adaptación es de Hernández, Suárez, Martínez-Tornisco y Hess en 1997 (Baena-Extremera \& Granero-Gallegos, 2013). Es un instrumento fiable, breve y de uso fácil para determinar la preocupación actitudinal sobre la calidad del medio ambiente y los cambios en las actitudes públicas y el impacto de políticas medioambientales, legislativas y del esfuerzo educativo (Weigel \& Weigel, 1978). La escala mide la preocupación ambiental en torno a la conservación y la contaminación del medio ambiente.

Para el análisis de las características sociales y culturales de los estudiantes, se utilizó un cuestionario que recoge la información socio demográfica. Los datos sociodemográficos 
son información que permite identificar características individuales y grupales de los individuos que participan en la investigación (Bonilla, 2019; Hernández-Sampieri, 2014; Hernández et al., 2018). Conocer estas características hizo posible relacionar la preocupación y el comportamiento ambiental con el conocimiento obtenido a través de la formación universitaria. De manera que la educación, edad, lugar de residencia, estatus social y económico, religión, característica familiar, son variables que pueden afectar la preocupación y el conocimiento sobre el ambiente. Esto repercute en la percepción de las consecuencias de los problemas ambientales y la disponibilidad de las personas para llevar a cabo acciones que contribuyen a solucionarlos (Corral-Verdugo \& Zaragoza, 2000). En base a las respuestas de los encuestados sobre los cursos que les proporciona formación ambiental se analizó el currículo de las carreras de formación en el centro universitario a fin de determinar los cursos específicos que contribuyen con el conocimiento ambiental.

\section{Población de estudio}

Los participantes de la investigación en la parte cuantitativa fueron los estudiantes de las carreras profesionales del CUNSURORI en el año 2020. Estas carreras son Agronomía, Zootecnia, Trabajo Social, Administración de Empresas, Ciencias Jurídicas y Sociales y Pedagogía, con una población estudiantil de $66,103,74,293,280$ y 229, respectivamente, para un total de 1,045 estudiantes. En la parte cualitativa se entrevistaron a los coordinadores y excoordinadores de las carreras, así como al director, asistente del director, coordinadora académica y representantes docentes y estudiantiles al Consejo Directivo.

El tema ambiental es importante en cada carrera, por ejemplo, Agronomía y Zootecnia, en su formación profesional se relaciona con el manejo sostenible de los recursos naturales y la sostenibilidad de los recursos naturales. La carrera de Pedagogía forma a los futuros docentes que favorecen el proceso de enseñanza- aprendizaje sobre el cuidado del 
ambiente. Trabajo Social trata de la organización de las personas, que es necesaria en el cuidado del ambiente. Administración de Empresas provee de profesionales para el manejo de los recursos humanos, físicos y financieros en la producción de riqueza que debe estar en equilibrio con el ambiente. La carrera de Derecho organiza, describe y establece toda la reglamentación jurídica y legal en el marco procesal, que es necesario en el resguardo medioambiental.

\section{Procedimiento}

La recolección de la información se realizó durante el año 2019 y 2020 , en los distintos ciclos de formación de las carreras indicadas. Las carreras que se encuestaron poseen diez ciclos académicos, que se distribuyen en un periodo de cinco años. La distribución anual de los ciclos es semestral, de manera que cada año se imparten dos ciclos. La recopilación de la información se realizó durante el segundo semestre del año 2019 y primer semestre de 2020, para la prueba piloto y la recolección de la información, respectivamente. En el año 2020, se encuestaron los ciclos primero, tercero, quinto, séptimo y noveno, para todas las carreras.

Por la pandemia de COVID-19 y suspensión de actividades algunos de los ciclos no fueron encuestados en su totalidad, pero se contó con la información necesaria para la tabulación de datos. La información se recopiló del total de la población estudiantil de las carreras, con los estudiantes presentes el día de la toma de datos. De manera que la presente investigación toma en cuenta el rigor científico, al tomar en cuenta que la ciencia se relaciona con aspectos observables y medibles; si las propuestas no involucran observación y pruebas públicas, no constituyen propuestas científicas (Kerlinger \& Howard, 2002). En la parte cualitativa se partió de un muestreo por conveniencia. Se tomó en cuenta la experiencia, opinión, jerarquía y perspectiva de los participantes para tomarlos en cuenta. Se entrevistó a autoridades académicas y administrativas, con las cuales se realizó una entrevista semiestructurada para conocer su enfoque sobre el tema ambiental que se imparte en el centro universitario. 
En la parte cuantitativa se inició la investigación con un análisis del instrumento, el cual fue validado a través de una prueba piloto practicada en el año 2019 con estudiantes de la carrera de Agronomía. Esta prueba permitió poder realizar ajustes a los ítems contenidos en los cuestionarios del instrumento de investigación, además de capacitar a estudiantes que sirvieron como apoyo para realizar las encuestas.

Para realizar la encuesta con los estudiantes de las carreras de Administración de Empresas, Agronomía, Ciencias Jurídicas y Sociales, Pedagogía, Trabajo Social y Zootecnia del centro universitario se realizó un muestreo aleatorio estratificado con asignación proporcional, donde se tomó una muestra de 402 estudiantes con un nivel de confianza del $95 \%$ y un grado de error de entre 2.3 y 3.5\%. En las primeras semanas del año 2020 se realizó la planificación de la realización de las encuestas, tomando en cuenta el tamaño de la muestra y se solicitaron las autorizaciones correspondientes con coordinación académica y los coordinadores de carrera.

Al realizar las encuestas, las mismas fueron codificadas para su organización y etiquetado. Los datos obtenidos de las encuestas fueron tabulados y sometidos a una limpieza y revisión para ser integrados en una hoja de cálculo y posteriormente analizar la información con pruebas estadísticas a través del programa SPSS. Se efectuaron las pruebas de normalidad por medio de momentos estadísticos y la prueba Kolmogorov-Smirnov (KS), luego se realizaron pruebas de fiabilidad a través del alfa de Cronbach y análisis factorial exploratorio por medio de la prueba Keyser Meyer Olkin (KMO). Se realizaron pruebas de diferenciación estadística con los datos aceptables como la prueba de medias de Tukey, Leve, Fruskal Wallis.

En la investigación cualitativa se realizó una muestra razonada con 7 coordinadores y excoordinadores de carrera y 5 autoridades administrativas. Se realizó el análisis del instrumento de entrevista, tomando 
en cuenta la revisión de tipos, momentos y efectos de la intervención dialogal, lo que permitió realizar ajustes entre los mismos. Se eligió la muestra razonada con coordinadores de carrera y autoridades administrativas. Los sujetos de investigación fueron caracterizados de acuerdo a su experiencia, conocimiento y puesto actual. Se realizó la reducción fenomenológica en base a conocimientos ambientales, contenidos curriculares en el tema ambiental, cursos, formación ambiental de docente y propuestas. Con lo anterior se procedió a realizar las entrevistas estructuradas, las cuales fueron transcritas para su análisis. Se revisaron y corrigieron las transcripciones para poder realizar la interpretación del discurso, lo cual se realizó por medio del programa NVIVO. Se crearon categorías para un análisis comparativo, las mismas fueron esquematizadas en sus componentes y relaciones, de lo que surgieron los temas producto del enfoque de diálogo. El análisis de dialogo tomó en cuenta las palabras más frecuentes, comparación de contrastes y similitudes entre participantes.

\section{Resultados}

Los datos obtenidos a través de las encuestas, se tabularon a través del programa MS Excel y luego se analizaron por SPSS. Se creó una base de datos con las respuestas de cada grupo de medición, es decir, de cada ciclo de las carreras en estudio. Con la información socio- demográfica se realizó una distribución de frecuencias; se visualizan las características de los estudiantes de las carreras. La información relevante indica que el $52 \%$ están comprendidos en edades de 16 a 20 años, el $52 \%$ son mujeres y el $48 \%$ son hombres, el $18 \%$ son solteros, el $93 \%$ se consideran ladinos, el 91\% pertenecen al área urbana, el $50 \%$ son católicos y el resto de otras religiones, el $56 \%$ proviene de centros educativos privados, el $53 \%$ obtienen sus ingresos de sus padres y el $34 \%$ trabajan.

Las escalas NEP-R y ECE fueron validadas en su contenido por docentes de la carrera de Agronomía del CUNSURORI, por una de las estudiantes del último año de ingeniería ambiental de la Facultad de Ingeniería de la USAC y por una de las docentes de 
dicha facultad. La validez aparente de dicha escala se realizó a través de una prueba piloto practicada con estudiantes de Agronomía del centro universitario, lo que permitió contextualizar algunos de los ítems. Se realizaron las pruebas de normalidad a la escala NEP-R a través de los momentos estadísticos de la media, moda, mediana y asimetría, también por la prueba de Kolmogorov-Smirnov (KS) y se obtuvo un nivel de significancia de 0.123 el cual es mayor de 0.05 lo que equivale que los datos de la escala en mención se comportan de forma Normal. En el caso de la escala ECE, se realizó la prueba KS y los datos presentaron una significancia de 0.00 lo que es menor a 0.05 , por lo que no se comportan de forma normal y no son factibles de análisis paramétrico.

La consistencia interna de los datos para las escalas se realizó por medio del alfa de Cronbach y correlaciones. Para evaluar la confiabilidad, fiabilidad o validez de un instrumento de medición es necesario el uso de algunos coeficientes como el desarrollado por J.L. Cronbach, el cual debe presentar valores entre 0.70 y 0.90 (Hernández-Sampieri, 2014). Al procesar la escala NEP-R se obtuvo un alfa de 0.668 , al eliminar los ítems 1, 7 y 15 que presentaron correlaciones muy bajas. Aquellos ítems que presentan correlaciones muy bajas pueden eliminarse. Así, Moyano y Palomo (Moyano-Diaz \& Palomo-Velez, 2014) indican que la confiabilidad puede mantenerse y es factible de eliminar de la prueba original, los ítems 1, 8, 11, 12; los autores de esta escala también recomiendan eliminar el ítem 16. En esta investigación los ítems 17 y 18 fueron adicionados por el autor y no son parte de la escala original. En el caso de los datos obtenidos, solamente el ítem 1 coincide con Moyano y Palomo. Vale la pena indicar que en la prueba piloto practicada inicialmente y eliminando los ítems propuestos por estos autores, el alfa de Cronbach que se obtuvo fue de 0.858. Una consistencia interna de datos aceptable debe presentar valores de alfa de Cronbach iguales o mayores de 0.70 (Cerda, et al., 2007). En cuanto a la escala ECE presentó valores de alfa de 0.671 al eliminar los ítems 1, 3, 7 y 10 que presentaron los menores valores de correlación. Respecto a la prueba piloto de esta escala la misma presentó un alfa de Cronbach inicialmente de 
0.885 , el cual se encuentra por arriba de 0.70 que requiere la confiabilidad de este coeficiente. Al tomar en cuenta la prueba de Keyser- Meyer- Olkin (KMO), donde la matriz anti- imagen muestra valores muy bajos de 1 y cercanos a 0 , para los ítems 1, 2, 3, 4 y 5, se realizó la prueba de confiabilidad sin considerar estos ítems, lo que mejoró los valores del alfa de Cronbach y se obtuvo un valor de 0.933 , lo cual no se reflejó con los datos finales de la investigación.

La validez es la estimación de la efectividad de los instrumentos de medición y determina si la acción de las variables independientes produce los efectos en las variables dependientes (Baena, 2017; Espinoza, 2010). En la validez del constructo el instrumento se mide por el grado en que una medición se relaciona consistentemente con otras mediciones sobre conceptos que están midiéndose (Bernal, 2006; GalludJardiel, 2015). Para la escala NEP-R dicha validez se realizó por medio de un análisis factorial exploratorio mediante la prueba Keyser- Meyer- Olkin (KMO) y la prueba Bartlett. El valor de la prueba KMO fue de 0.681 con la correlación de 9 ítems y se obtuvieron 4 factores con valores arriba de 0.5 , en lo que se coincidió con varios autores como Vozmediano y San-Juan (2005) también con Amérigo y González (2001), quienes realizaron investigaciones sobre las propiedades psicométricas de la escala NEP-R en diferentes muestras encontraron que el análisis factorial extrajo los mismos 4 factores como lo son antropocentrismo, ecocentrismo, limitaciones de la biosfera y confianza del ser humano ante la naturaleza. En la escala ECE el valor de KMO fue de 0.744 con 14 ítems y se obtuvieron 5 factores con valores por arriba de 0.5. Al realizar un análisis teórico comparativo sobre otras investigaciones realizadas en el mismo tema, se coincidió con lo reportado por Amérigo y González en 1996 (BaenaExtremera \& Granero-Gallegos, 2013) quienes encontraron 5 factores que conforman la escala y con Aragonés \& Amérigo (1991) que encontraron 4 componentes. Los cinco factores en que se conforma la escala son el esfuerzo personal para conservar, contaminación industrial, control no personal, prevención institucional y el activismo ambiental. 
Se realizó el análisis de varianza para la escala NEP-R con la finalidad de encontrar diferencias entre los ciclos de formación de las diferentes carreras y se obtuvo un valor de significancia de 0.032 lo que es menor de 0.05 . Por lo que existen diferencias entre los ciclos. Para encontrar diferencias entre los datos, se realizaron pruebas de medias que evalúan si los datos de dos grupos tienen diferencias significativas con respecto a sus medias en la variable que se elija (Flores, 2012), por lo cual se realizó la prueba de medias de Tukey y se determinó que las diferencias significativas se encuentran entre el tercero y quinto ciclo de formación de las carreras. Sobre la escala ECE, por no tener datos normales, se realizó la prueba no paramétrica de Kruskal Wallis para encontrar diferencias entre los ciclos y se obtuvo una significancia asintótica de 0.029 lo que es menor a 0.05 , lo que indica que existen diferencias; estas se presentan entre el quinto y séptimo ciclo de las carreras del centro universitario.

Al analizar la información proporcionada por los estudiantes respecto al contenido ambiental de los cursos en sus carreras, el $56 \%$ opinaron que no tienen dichos contenidos. Al solicitar información sobre el nombre de los cursos con contenidos ambiental, únicamente las carreras de Agronomía y Zootecnia mencionaron algunos. En estas dos carreras los estudiantes indican que su conocimiento ambiental es producto de su formación universitaria en un 75 y $57 \%$, respectivamente. Se analizaron los primeros cuatro cursos que les proporcionan conocimiento ambiental en cada carrera, en opinión de los estudiantes se determinó que corresponde al 16 y $11 \%$, respectivamente. Sin embargo, al realizar el análisis de contenido curricular, se encontró que dichos cursos, en Agronomía y Zootecnia poseen en promedio 78 y $64 \%$ de contenido ambiental, respectivamente.

En la investigación cualitativa se realizó una categorización sobre los temas más consistentes en el dialogo con los participantes, los cuales fueron sobre los docentes y el tema ambiental, propuestas de formación en temas ambientales, capacitación docente y la política ambiental. Los diálogos de las entrevistas se pueden resumir según el análisis de 
frecuencia de palabras en la siguiente oración, "Los temas ambientales en los cursos debe considerar la capacitación del trabajo docente en relación a la política". Se realizó análisis de elementos conglomerados por similitud de codificación, donde las mayores diferencias de opinión se encontraron entre el coordinador de administración de empresas y la asistente de dirección con el representante estudiantil ante el Consejo Directivo.

\section{Conclusiones}

Se caracterizaron sociodemográficamente a los estudiantes entrevistados, los cuales se encuentran en edades de 16 a 20 años, equilibrados en cuanto al sexo, son ladinos, originarios de Jalapa, provienen de centros educativos privados. Se practicaron dos escalas para determinar el comportamiento ecológico (NEP-R) la preocupación ambiental (ECE). Se determinó que existen diferencias entre los ciclos de formación de las carreras. Sin embargo, al considerar las diferencias que se obtienen al ingreso y egreso de los estudiantes a la universidad, las diferencias no son significativas. Por tanto, la formación universitaria no influye sobre el comportamiento ecológico y la preocupación ambiental en sus estudiantes. Al indagar en los estudiantes si el conocimiento ambiental es producto de su formación universitaria, únicamente las carreras de Agronomía y Zootecnia presentan porcentajes del 75 y $57 \%$. Empero, en términos generales las carreras del CUNSURORI en un $56 \%$ no se les imparte cursos con contenido ambiental. Al analizar los contenidos curriculares de los cursos que en opinión de los estudiantes les favorecen en su formación ambiental, las carreras de Agronomía y Zootecnia poseen dichos contenidos en porcentajes favorables. En relación a la importancia que le proveen los responsables a contenidos y actividades ambientales, es mínima. Producto del análisis de diálogo coinciden que debe prestársele atención a la docencia en temas ambientales, propuestas de formación, capacitación de docentes y la política ambiental. 
Las autoridades deben integrar el contenido ambiental en los cursos de las carreras para que los índices de comportamiento ecológico y la preocupación ambiental aumente en los años de formación. Proveer en diferentes cursos de formación en las carreras del CUNSURORI de los temas ambientales relacionados a la profesión para contribuir a disminuir el deterioro actual del ambiente. Tomar en cuenta la implementación de la enseñanza ambiental en la actualización periódica de programas de estudio en cada ciclo de formación. En los rediseños curriculares considerar la enseñanza e implementación de la educación de ambiente incluyendo la política ambiental de la USAC y otras normas relacionadas. Evaluar los cursos de contenido ambiental en las carreras que los poseen para hacer coincidir la percepción del contenido ambiental de los estudiantes con el contenido ambiental de los cursos que reciben. Crear una comisión de Ambiente del CUNSURORI para proponer proyectos y acciones a implementar en la enseñanza ambiental. 


\section{Referencias}

Acosta-Martínez, J., Montero, M., \& López-Lena, L. (2001).

Relación entre conducta proambiental y algunos componentes psicológicos en estudiantes mexicanos.

Medio Ambiente y Comportamiento Humano, 2(1), 45-58. http://mach.webs.ull.es/PDFS/VOL2_1/ Vol_2_1_c.pdf

Alvarez, P., \& Vega, P. (2009). Actitudes ambientales y conductas sostenibles. Implicaciones para la educación ambiental. Revista de Psicodidáctica, 14(2), 245-260. https://www.redalyc.org/ pdf/175/17512724006.pdf

Amérigo, M.; González, A. (2001). Los valores y las creencias medioamentales en relación con las decisones sobre dilmeas ecológicos. Estudios de Psicología, 22(1), 65-73. https://dialnet.unirioja.es/servlet/ articulo?codigo $=66227$

Aragonés, J., \& Amérigo, M. (1991). Un estudio empírico sobre las actitudes ambientales. Revista de Psicología Social, 6(2), 223-240. https://doi.org/10.1080/02134 748.1991 .10821647

Baena-Extremera, A., \& Granero-Gallegos, A. (2013). Estudio cuasi- esperimental de un programa de supervivencia en el medio natural. Revista Internacional de Medicina y Ciencias de La Actividad Física y El Deporte, 13(51), 551-567. http://cdeporte.rediris.es/revista/ revista51/artestudio384.pdf

Baena, G. (2017). Metodología de la investigación (J. Enrique (ed.); Tercera ed). Grupo editorial Patria. https://hopelchen.tecnm.mx/principal/sylabus/fpdb/ recursos/r131102.PDF

Bernal, C. (2006). Metodología de la investigación. Para administración, economía, humanidades y ciencias sociales (Segunda ed). Pearson Educación. 
Best, J. (1982). Como investigar en educación (Novena edi). Ediciones Morata, S.A.

Bifani, C. (1984). Desarrollo y medio ambiente. Publicaciones del Ministerio de Obras Públicas y Urbanismo.

Bonilla, G. (2019). Investigación científica; Métodos, técnicas e instrumentos. Editorial Servi prensa.

Briones, G. (1996). Metodología de la investigación cuantitativa en las ciencias sociales. (Icfes (ed.)). Instituto colombiano para el fomento de la educación superior, ICFES. https://books.google.com.gt/books/ about/Metodología_de_la_investigación_cuanti. html?id=su2YtgAACAAJ\&redir_esc=y

Cerda, A., García, L., Díaz, M., \& Nuñez, C. (2007). Perfil y conducta ambiental de los estudiantes de la Universidad de Talca, Chile. Panorama Socioeconómico, 25(35), 148-159. https://www. academia.edu/47091113/Perfil_de_la_conducta_ ambiental_de_los_alumnos_de_la_Universidad_de_ Talca

COBAO. (2017). Metodología de la investigación. Guía para el autoaprendizaje (A. Martínez, B. Méndez, E. Osorio, M. Garcia, \& R. Toledo (eds.); Primera ed). Sistema de educación abierta -SEA-. http://www.cobao.edu. $\mathrm{mx} /$ images/PDF/CEA/Cuatrimestre2/metodologiaSEA-10-2017.pdf

Corona, J. (2016). Apuntes sobre métodos de investigación. Medisur, 14(1), 81-83. http://scielo.sld.cu

Corral-Verdugo, V., \& Zaragoza, F. (2000). Bases sociodemográficas y psicológicas de la conducta de reuitilización: un modelo estructural. Medio Ambiente y Comportamiento Humano, 1(1), 9-29. https:// investigadores.unison.mx/es/publications/basessociodemográficas-y-psicológicas-de-la-conducta-dereutiliz-2 
Espinosa, Y. (2015). Caracterización de los comportamientos humanos que propician cambios educativos y su incidencia en el aprendizaje organizacional [Tecnológico de Montorrey]. https://repositorio.tec.mx/bitstream/ handle/11285/621308/02Yuly Caterine Espinosa Pineda.pdf?sequence $=1$ \&isAllowed $=y$

Espinoza, C. (2010). Metodología de investigación tecnológica. Pensando en sistemas (C. Espinoza (ed.); Primera ed). Imagen Grafica SAC. https://ciroespinoza. files.wordpress.com/2012/01/metodologc3ada-deinvestigacic3b3n-tecnolc3b3gica.pdf

Flores, R. (2012). Investigación en educación ambiental.

Revista Mexicana de Investigación Educativa, 17(55), 1019-1033. https://www.redalyc.org/ pdf/140/14024273002.pdf

Gallud-Jardiel, E. (2015). Manual practico para escribir una tesis (Primera ed). Editorial Verbum, S.L. https:// www.marcialpons.es/libros/manual-practico-paraescribir-una-tesis/9788490742051/

Guarisma, J. (2008). El comportamiento educativo. Revista Iberoamericana de Educacón, 2(47), 2. https://rieoei. org/RIE/article/view/2369

Gutiérrez, M. (1992). La percepción en la Filosofía de Alfred Ayer. Ideas y Valores, 87-88, 14. https://es.scribd. com/document/253160972/Gutierrez-MalaverMaria-Elsa-La-Percepcion-en-La-Filosofia-de-AlfredAyer

Hernández-Sampieri, R. (2014). Metodología de la Investigación (M. Rocha (ed.); Sexta Edic). Mac Graw Hill education. https://periodicooficial.jalisco.gob. $\mathrm{mx} /$ sites/periodicooficial.jalisco.gob.mx/files/ metodologia_de_la_investigacion_-_roberto_ hernandez_sampieri.pdf 
Hernández, A., Ramos, M., Placencia, B., Idacochea, B., Quimis, A., \& Moreno, L. (2018). Metodología de la investigación científica. Editorial Area de Innovación y Desarrollo, S.L. https://doi.org/http://dx.doi. org/10.17993/CcyLI.2018.15

Isaac-márquez, R., Salavarría, O., Eastmond, A., Ayala, M., Arteaga, M., Isaac-Márquez, A., Sandoval, J., \& Manzanero, L. (2011). Cultura ambiental en estudiantes de bachillerato . Estudio de caso de la educación ambiental en el nivel medio superior de Campeche. Revista Electrónica de Investigación Educativa, 13(2), 100. http://redie.uabc.mx/vol13no2/contenidoisaacmarquezetal.html

Kerlinger, F., \& Howard, L. (2002). Investigación del comportamiento. Métodos de investigación en ciencias sociales (Mc Graw Whill Ineramericana Editores (ed.); Cuarta edi). Mc Graw Hill. https://padron.entretemas. com.ve/INICC2018-2/lecturas/u2/kerlingerinvestigacion.pdf

Mata-Segreda, A. (2004). Transformación de la cultura ambiental mediante la docencia universitaria. Revista Biocenosis, 129-135. https://www.researchgate. net/publication/242401662_Transformacion_de_ la_cultura_ambiental_mediante_la_docencia_ universitaria

Mendoza, J. \& Garza, J. (2009). La medición en el proceso de investigación científica: evaluación de validez de contenido y confiabilidad. Innovaciones de Negocios, 6(1), 17-32. http://eprints.uanl.mx/12508/

Miranda, L. (2013). Cultura ambiental: un estudio desde las dimensiones de valor, creencias, actitudes y comportamientos ambientales. Producción Mas Limpia, 8(2), 94-105. http://www.scielo.org.co/scielo. php?pid=S1909-04552013000200010\&script $=$ sci_ abstract\&tlng=es 
Motta, C. (1994). La formación y la vivencia de los valores en las escuelas costarricenses. Ministerio de educación Pública - UNESCO. https://www.worldcat.org/title/ formacion-y-la-vivencia-de-los-valores-en-lasescuelas-costarricenses/oclc/34278105

Moyano-Diaz, E., \& Palomo-Velez, G. (2014). Propiedades psicométricas de la Escala Nuevo Paradigma Ecológico (NEP-R) en poplación chilena. Psicología Ambiental, 45(3), 415-423. https://www.researchgate. net/publication/279174399_Propiedades_ Psicometricas_de_la_Escala_Nuevo_Paradigma_ Ecologico_NEP-R_en_Poblacion_Chilena

Navarro, E., Jiménez, E., Rappoport, S., \& Thoilliez, B. (2017). Fundamentos de la investigación y la innovación educativa (Primera ed). Universidad Internacional de la Rioja, S.A. https://www.unir.net/wp-content/ uploads/2017/04/Investigacion_innovacion.pdf

Páramo, P. (2016). Reglas proambientales: una alternativa para disminuir la brecha entre el decir-hacer en la educación ambiental. Suma Psicológica, 4, 1-17. https://doi.org/10.1016/j.sumpsi.2016.11.001

Piloña, G. (2005). Guía práctica sobre métodos y técnicas de investigación documental y de Campo. (G. Editores (ed.); Sexta edic). Editorial CIMGRA. https://isbn. cloud/9789992297506/guia-practica-sobremetodos-y-tecnicas-de-investigacion-documental-yde-campo/

Ramos, C. (2015). Los paradigmas de la investigación científica. Av. Psicol., 23(1). http://www.unife.edu.pe/ publicaciones/revistas/psicologia/2015_1/Carlos_ Ramos.pdf

Reyes, A. (1959). Comportamiento educativo. Revista de Educación - Estudios, 32(91), 35-38.

Sanz, L., \& Guillén, C. (2005). Escala nuevo Paradigma Ecológico: propiedades psicométricas con una muestra española obtenida a través de Internet. Medio 
Ambiente y Comportameinto Humano, 6(1), 37-49. https://mach.webs.ull.es/PDFS/Vol6_1/VOL_6_1_d. pdf

Tamayo, M. (2007). El proceso de la investigación científica (E. Limusa. (ed.); 4a. edició). http://evirtual.uaslp.mx/ ENF/220/Biblioteca/Tamayo Tamayo-El proceso de la investigación científica2002.pdf

Tyler, R. (1986). Principios básicos del currículo. Troquel.

Uzzell, D., \& Rathzel, N. (2009). Transformando la psicología ambiental. Journal of Envirnmental Psychology, 29(3), 340-350. https://doi.org/10.1016/j.jenvp.2008.11.005

Velásquez, C. (2017). La investigación cientifica, un acercamiento didáctico (Segunda). ECO ediciones. https://isbn.cloud/9789929694057/la-investigacioncientifica/

Vozmediano, L., \& San-Juan, C. (2005). Escala Nuevo Paradigma Ecológico: propiedades psicométricas con una meustra española obtenida a través de Internet. Medio Ambiente y Comportameinto Humano, 6(1), 37-49. https://mach.webs.ull.es/PDFS/Vol6_1/ VOL_6_1_d.pdf

Weigel, R., \& Weigel, J. (1978). environmetal concern:

The deveolpment of a measure. Environment and Behavior, 10(1), 3-15. https://doi. org/10.1177\%2F0013916578101001

Zapata, R., \& Castrechini, A. (2011). Conducta proambiental y personalidad: analísis de un barrio de Lima. Quaderns de Psicología, 13(1), 47-61. https:// www.researchgate.net/deref/http\%3A\%2F\%2Fwww. quadernsdepsicologia.cat\%2Farticle\%2Fview\%2F932 


\section{Semblanza académica del autor}

Profesor Titular del Centro Universitario de Sur Oriente -CUNSURORI- de la Universidad de San Carlos de Guatemala -USAC-, con sede en la ciudad de Jalapa. Con estudios a nivel de licenciatura en Agronomía y Administración de empresas. Graduado de la Maestría en Docencia Universitaria y del Doctorado en Educación en la USAC. Es Coordinador de la Práctica Profesional Supervisada -PPS- de la carrera de Agronomía en el CUNSURORI. Ha producido texto de apoyo a la docencia como Introducción al estudio de los nombres de las plantas y Descripción taxonómica del grupo de plantas: Pinophytas y Magnoliophytas. Además de realizar investigaciones y publicaciones en el tema ambiental. 